\title{
Compliant actuation based on dielectric elastomers for a force-feedback device: modeling and experimental evaluation
}

\author{
R. Vertechy, M. Bergamasco \\ PERCRO laboratory, Scuola Superiore Sant'Anna, Pisa, Italy \\ r.vertecby@sssup.it,m.bergamasco@sssup.it \\ G. Berselli \\ Department of Engineering "Enzo Ferrari"- University of Modena and Reggio Emilia, Modena, Italy \\ Italygiovanni.berselli@unimore.it
}

V. Parenti Castelli, G. Vassura

Department of Mechanical and Aeronautical Engineering, University of Bologna, Bologna, Italy vincenzo.parenti@unibo.it,gabriele.vassura@unibo.it

\begin{abstract}
Thanks to their large power densities, low costs and shock-insensitivity, Dielectric Elastomers (DE) seem to be a promising technology for the implementation of light and compact force-feedback devices such as, for instance, haptic interfaces. Nonetheless, the development of these kinds of DE-based systems is not trivial owing to the relevant dissipative phenomena that affect the DE when subjected to rapidly changing deformations. In this context, the present paper addresses the development of a force feedback controller for an agonist-antagonist linear actuator composed of a couple of conically-shaped DE films and a compliant mechanism behaving as a negative-rate bias spring. The actuator is firstly modeled accounting for the viscohyperelastic nature of the DE material. The model is then linearized and employed for the design of a force controller. The controller employs a position sensor, which determines the actuator configuration, and a force sensor, which measures the interaction force that the actuator exchanges with the environment. In addition, an optimum full-state observer is also implemented, which enables both accurate estimation of the time-dependent behavior of the elastomeric material and adequate suppression of the sensor measurement noise. Preliminary experimental results are provided to validate the proposed actuator-controller architecture.
\end{abstract}

KEYWORDS. Dielectric elastomers; Agonist-antagonist actuation; Force-feedback control; Haptic interfaces.

\section{INTRODUCTION}

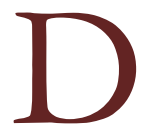

ielectric Elastomer (DE) films are visco-elastic capacitors which experience deviatoric deformations and/or generate forces when subjected to high electric potential (voltage) differences [1,2]. Thanks to the large force and power densities, relevant compliance and damping, and low effective inertia and cost, DE actuators are a promising technology for the development of affordable mechatronic and robotic systems that have to interact effectively, efficiently and safely with unstructured environments and humans [3]. In particular, as already demonstrated by several proof-of-concept prototypes developed in different research institutes all over the world, DE actuators can be profitably used for the realization of practical force feedback devices such as, for instance, Haptic Interfaces (HI) for immersive virtual reality [4,5]. HI are mechatronic devices capable of modulating the forces exchanged with a human operator in 
order to provide a global sensations of kinesthetic type or local sensations of tactile type. Possible advantages that HI can offer to the society come from the realization of the following systems:

$\checkmark$ Simulation in virtual environments for training people to a specific activity (for instance, medical diagnoses and surgical interventions);

$\checkmark$ Prototyping by computer aided design (CAD), where the artist or the designer could use the HI for touching or handling the object or the part they are working on;

$\checkmark$ Teleoperation from a remote position for the execution of delicate tasks in hazardous or hardly accessible environments;

$\checkmark$ Multimodal interaction, by which also disable users (for instance, blinds people) could remotely communicate with personal computers and the net more extensively.

Nowadays, the availability and diffusion on a large scale of the aforementioned systems is limited as long as existing HI are frequently expensive, difficult to manufacture, assemble and maintain, and characterized by low payload to weight ratio and shock sensitivity. Within this scenario, the use of smart materials such as DE could pave the way to the realization of non-conventional actuation systems with suitable performances to build better-behaved HI, characterized by large power densities, low costs and shock-insensitivity. Nonetheless, these DE-based environment-interacting devices require stable, fast and accurate regulation of the exchangeable force. This task can actually become quite challenging owing to the relevant dissipative phenomena that affect the majority of DE materials when subjected to rapidly changing deformations.

As a first step towards the production of practical DE-based force feedback devices and HI, the present paper addresses the development of a force controller for an agonist-antagonist linear actuator (see Fig. 1). The actuator quasi-static response is predicted on the basis of a non-linear model previously proposed by the authors [6]. Then, the system timedependent behaviour is identified resorting to the well-known Quasi-Linear Viscoelastic (QLV) model [7,8], frequently adopted with the sake of compromising between the simplicity of classical linear theories and the difficulty of nonlinear approaches. The overall actuator model is then linearized and employed for designing a force controller which employs a position sensor, is closed around a custom-made force sensor (measuring the actuator-environment interaction force), and implements a state-feedback control law and a Kalman filter [9]. This optimum full-state observer makes it possible to compensate for intrinsic DE hysteresis and stress relaxation, and to clean-out the sensor measurement noise that usually degrades controller performance. At last, the force regulation capability of the DE actuator-controller system is evaluated in dynamic conditions via a properly predisposed experimental test-bench.

\section{DESCRIPTION OF THE ACTUATOR PROTOTYPE}

$\checkmark$ he actuator CAD model is depicted in Fig. 1 and comprises:

$\checkmark$ A rigid frame made by two coaxial identical rings with internal radii equalling $r_{M}=40 \mathrm{~mm}$ and connected by four rods with lengths equalling $2 d=40 \mathrm{~mm}$;

$\checkmark$ An over-constrained compliant parallel mechanism featuring a rigid circular moving platform with external radius equalling $r_{m}=12 \mathrm{~mm}$. The mechanism pseudo-rigid body model [10] is depicted in Fig. 2 . The platform is connected to one of the rigid frame rings via three symmetrically-located identical legs, each articulated via three revolute elastic joints having parallel axes.

$\checkmark$ Two conically-shaped DE films (film \#1 and film \#2) connecting the two rigid frame rings to the mechanism platform (i.e. the actuator output) in an agonist-antagonist arrangement.

As previously described in [6], the over-constrained compliant parallel mechanism behaves as a negative stiffness (bias) spring. In particular, thanks to the employed architecture, the actuator output can only move along the actuator axial direction (i.e. the axis of symmetry of the two rigid frame rings). Therefore, each leg of the parallel mechanism behaves as a compliant eccentric slider-crank mechanism (Fig. 3) with eccentricity, crank and connecting-rod lengths equal to $e=32 \mathrm{~mm}, r_{c}=34.5 \mathrm{~mm}$ and $r_{r}=21.2 \mathrm{~mm}$, and elastic joint torsional stiffnesses and undeflected angular positions equal to $k_{1}=k_{2}=1 \mathrm{mNm} / \mathrm{rad}, k_{3}=51 \mathrm{mNm} / \mathrm{rad}, \vartheta_{c 0}=26^{\circ}, \vartheta_{c 0-r 0}=258^{\circ}$ and $\vartheta_{p 0}=232^{\circ}$.

Each DE film is a circular membrane of acrylic elastomer (VHB-4905 by 3M) with initial thickness (in its undeformed state) equalling $t_{0}=1.5 \mathrm{~mm}$, subjected to an equibiaxial pre-stretch equalling $\lambda_{p}=4$, and coated with a pair of compliant carbon conductive grease electrodes. Prior to their use, these virgin DE membranes are subjected to preconditioning loading-unloading cycles (as in [6]), which yields a residual stretch (permanent set, $\lambda_{r}=1.6$ ) whose value has been 
estimated on sacrificial specimen. A voltage difference $V_{1}\left(V_{2}\right)$ between the electrode pair of the DE film \#1 (\#2) generates a rightward (leftward) force on the actuator output. The bias spring has been designed so that the overall actuator in its OFF-state mode (i.e. $V_{1}=V_{2}=0$ ) possesses a rest (stable) position when the actuator output lies exactly in the middle of the rigid frame rings (i.e. when $x=d$ ). Also, the actuator maintains a positive stiffness across the desired stroke (see Sec. Mathematical model of the dielectric elastomer film force). For each actuator output position, reciprocal activation of the agonist-antagonist DE films enables feed-forward independent regulation of the actuator interaction force.

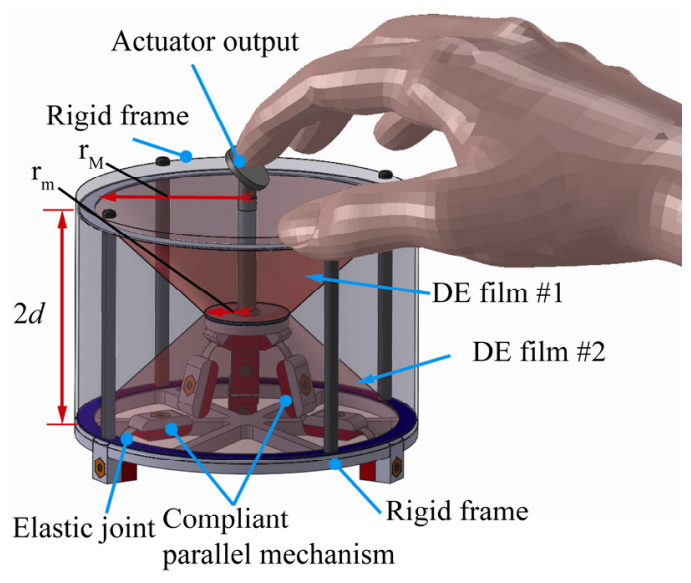

Figure 1: DE actuator CAD model.

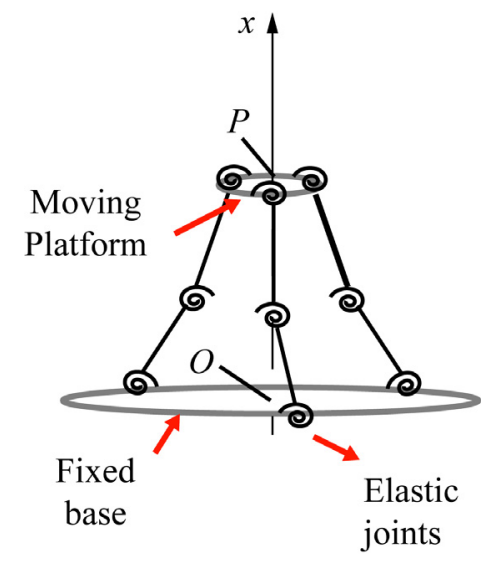

Figure 2: Parallel compliant mechanism. Pseudo-rigid model.

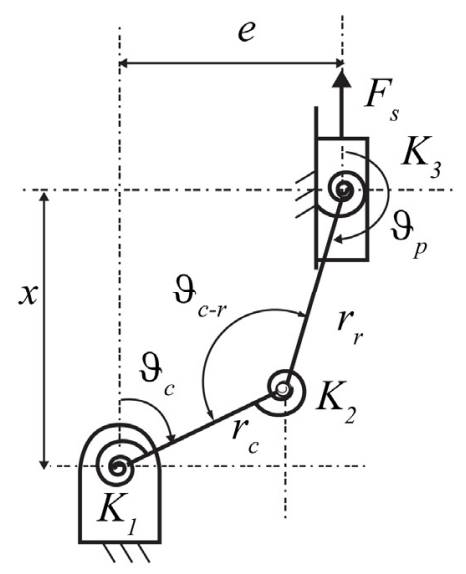

Figure 3: Slider-crank schematic.

\section{MATHEMATICAL MODEL OF THE DIELECTRIC ELASTOMER FILM FORCE}

$\mathrm{L}$

et first consider the DE film \#1. The expression of the overall external force, $F_{f(1,1)}$, that must be supplied at $\mathrm{O}$ and $\mathrm{P}$ (and directed along the line joining these points, Fig. 2) to balance the DE internal reaction force at a given generic configuration $\mathrm{x}$ of the actuator, can be split as:

$$
F_{f, 1}\left(x, \dot{x}, V_{1}\right)=F_{v e, 1}(x, \dot{x})-F_{e m, 1}\left(x, V_{1}\right)
$$

where $F_{(p, 1)}$ represents the viscoelastic response of the DE film and $F_{(e m, 1)}$ represents an electrically induced term, having the dimension of a force and usually referred to as Maxwell force [11,12].

As for the electrically induced force, a suitable expression for conically-shaped DEs has been derived in $[6]$ and it is given by:

$$
F_{e m, 1}\left(V_{1}, x\right)=\chi x \pi^{2} V_{1}^{2}\left(r_{M}+r_{m}\right)^{2} \varepsilon / v
$$


where $v=\pi\left(r_{M}^{2}-r_{m}^{2}\right) t_{0} \lambda_{p}^{-2}$ is the DE volume, $\varepsilon=4.5 \cdot 8.85 e-12 F / m$ is the dielectric permittivity of the acrylic film, and $\chi=0.6$ is a suitable dimensionless correction factor. This expression is based on the assumption that the incompressible DE is a right circular conical horn with constant wall thickness in any of its deformed configurations.

As for the DE viscoelastic response, a possible approach is to consider the force response due to a step change in displacement and to superimpose each contribution of a displacement history, $x(t)$, by applying a proper superposition principle. Resorting to a one dimensional model, the overall force response is then given by:

$$
F_{v e, 1}(t)=\int_{0}^{t} \hat{\mathcal{K}}(t-\tau) \cdot d[x(\tau)]=\int_{0}^{t} \hat{\mathcal{K}}(t-\tau) \frac{d x(\tau)}{d \tau} d \tau
$$

having assumed $x=0$ for $t<0$ and a differentiable displacement history. The function $\hat{\mathcal{K}}(t, x)$ is named relaxation function and specifies the force response to a unit step change in displacement. In the QLV framework [7,8], the relaxation function takes the form:

$$
\hat{\mathcal{K}}(x, t)=F_{e, 1}(x) \cdot g(t) \quad \text { with } \quad g(0)=1
$$

where $F_{(e, 1)}(x)$ is the elastic response, i.e. the force generated by an instantaneous displacement, whereas $g(t)$, called reduced relaxation function, describes the time-dependant behavior of the material. As for the latter term, it is customary to assume a linear combination of exponential functions, the exponents $v_{i}$ identifying the rate of the relaxation phenomena, and the coefficients $c_{i}$ depending on the material:

$$
g(t)=\sum_{i=0}^{r} c_{i} e^{-v_{i} \cdot t} \quad \text { with } \quad \sum_{i=0}^{r} c_{i}=1
$$

where, in general, $v_{0}=0$. Finally, the total force at the instant $t$ is the sum of the contributions due to all past changes [13], i.e.

$$
\begin{aligned}
& F_{v e, 1}(t)=\int_{0}^{t} g(t-\tau) \frac{\partial F_{e, 1}[x(\tau)]}{\partial x} \frac{\partial x(\tau)}{\partial \tau} d \tau \\
& =\int_{0}^{t} g(t-\tau) K_{e, 1}[x(\tau)] \dot{x}(\tau) d \tau
\end{aligned}
$$

where $K_{e, 1}(x)=\partial F_{e, 1}[x] / \partial x$. By substituting Eqs. (4) and (5) in Eq. (7), one obtains:

$$
F_{v e, 1}(t)=\int_{0}^{t} K_{e, 1}(x) \cdot\left[c_{0}+\sum_{i=1}^{r} c_{i} e^{-v_{i}(t-\tau)}\right] \cdot \dot{x}(\tau) d \tau
$$

In particular, referring to Fig. 4, the force response given by the QLV model can be interpreted as that of a nonlinear stiffness connected by a series of $r$ linear Kelvin models (i.e. a parallel spring-damper system).

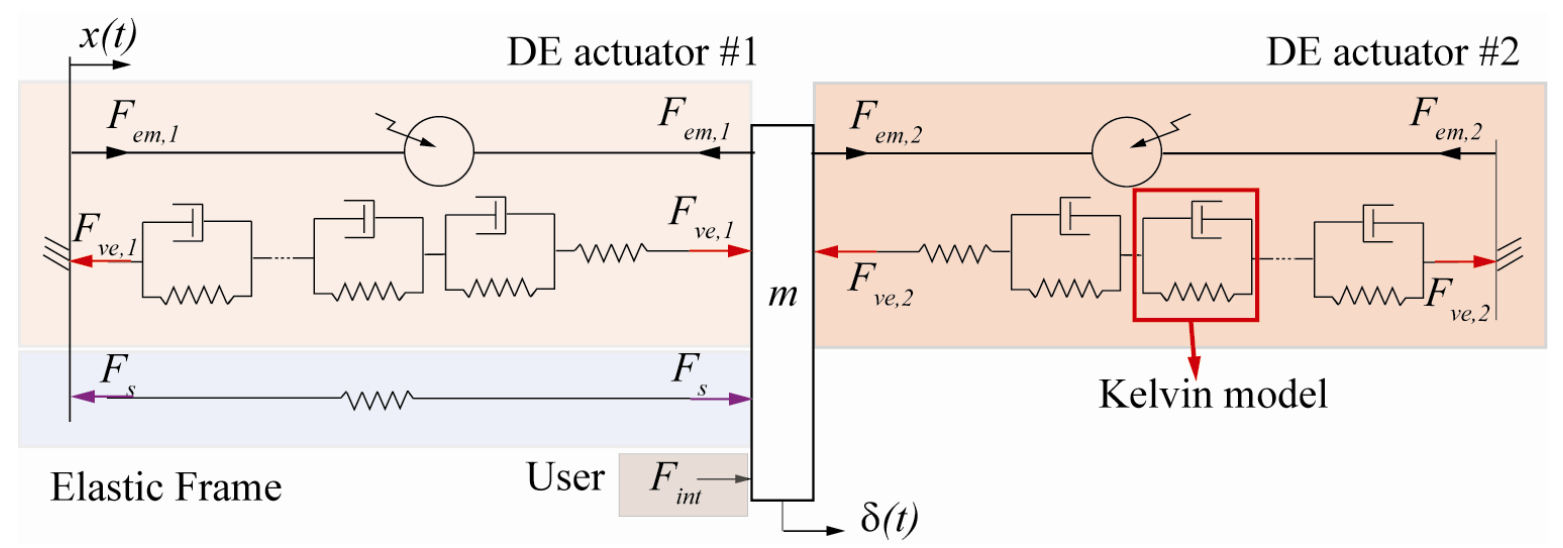

Figure 4: Actuator non-linear lumped parameter model. 
Concerning the DE quasi-static response, a suitable expression for the elastic force of conically-shaped DEs has been derived in [6] and it is given by:

$$
\left.F_{e, 1}(x)=\xi v\left[\sum_{i=1}^{3} i C_{i}\left(\lambda_{1}^{2}+\lambda_{2}^{2}+\lambda_{1}^{-2} \lambda_{2}^{-2}-3\right)^{i-1}\right] \cdot \frac{\left(\lambda_{1} \lambda_{2}-\lambda_{1}^{-3} \lambda_{2}^{-1}\right) x}{\left[\left(r_{M}-r_{m}\right)\right) \sqrt{x^{2}+\left(r_{M}-r_{m}\right)^{2}}}\right]
$$

where $\lambda_{2}=\lambda_{p} / \lambda_{r}$ and $\lambda_{1}=\lambda_{2} \sqrt{1+x^{2} /\left(r_{M}-r_{m}\right)^{2}}$ are the longitudinal and latitudinal stretches of the DE middle surface, $C_{1}=30488 \mathrm{~Pa}, C_{2}=151 \mathrm{~Pa}, C_{3}=8 \mathrm{~Pa}$ are $\mathrm{DE}$ constitutive parameters of a Yeoh-type hyperelastic strain-energy function [14], and $\chi=0.93$ is a dimensionless correction factor. Concerning the reduced relaxation function, $r=3$, $c_{0}=0.83, c_{1}=0.22, c_{2}=1-c_{1}-c_{0}, v_{1}=4.30 \mathrm{~s}^{-1}, v_{2}=0.70 \mathrm{~s}^{-1}$.

At last, the contribution of the compliant frame, $F_{s}(x)$ can be easily evaluated resorting to the pseudo-rigid-body approximation (Figs. 2 and 3). In particular, the following relationships are found from the position analysis of a single slider-crank mechanism:

$$
\vartheta_{p}=\pi-\operatorname{asin}\left(\frac{r_{c} \sin \left(\vartheta_{c}\right)-e}{r_{r}}\right) ; \quad \alpha=\operatorname{atan}\left(\frac{e}{x}\right) ; x=r_{c} \cos \left(\vartheta_{c}\right)-r_{r} \cos \left(\vartheta_{p}\right)
$$

From the static analysis of the overall compliant frame having three equal legs, the following equation holds:

$$
F_{s}=\frac{3 K_{1} \Psi_{1} \cos \left(\vartheta_{p}\right)}{r_{c} \sin \left(\vartheta_{p}-\vartheta_{c}\right)}+\frac{3 K_{2} \Psi_{2} \cos (\alpha)}{r_{c} \sin \left(\vartheta_{c}-\alpha\right)}+\frac{3 K_{3} \Psi_{3} \cos \left(\vartheta_{c}\right)}{x \sin \left(\vartheta_{c}\right)-e \cos \left(\vartheta_{c}\right)}
$$

Where $\Psi_{1}=\vartheta_{c}-\vartheta_{c 0}, \Psi_{2}=\vartheta_{p}-\vartheta_{p 0}-\vartheta_{c}+\vartheta_{c 0}, \Psi_{3}=\vartheta_{p}-\vartheta_{p 0}$.

Concerning the agonistic-antagonistic actuator, denoting $\delta$ as the actuator output position measured from the OFF-state rest location along its axial direction $x=d$ (hereafter this location is referred to as actuator central position), the overall actuator force will be given by:

$$
F_{f, 1-2}\left(\delta, \dot{\delta}, V_{1}, V_{2}\right)=F_{f, 1}\left(d+\delta, \dot{\delta}, V_{1}\right)-F_{f, 2}\left(d-\delta, \dot{\delta}, V_{2}\right)+F_{s}(d+\delta)
$$

where, with obvious notation, $F_{f, 2}=F_{v e, 2}-F_{e m, 2}$ is the reaction force of the DE film \#2.

In particular, Figs. 5 and 6 report the simulated Force-Position (FP) curves of the prototype actuator for the voltage sets $\left\{V_{1}=0, V_{2}=0\right\}$ (solid line), $\left\{V_{1}=6.7 \mathrm{kV}, V_{2}=0\right\}$ (circle marks) and $\left\{V_{1}=0, V_{2}=6.7 \mathrm{kV}\right\}$ (dot marks), for two sinusoidal trajectories with $10 \mathrm{~mm}$ amplitude but different frequencies equalling $1 \mathrm{mHz}$ and $0.5 \mathrm{~Hz}$ respectively. These plots highlight that, within the considered range of motion, the quasi-static response of the considered DE actuator is rather elastic and linear, whereas its dynamic behavior is severely affected by the hysteresis of the acrylic elastomeric material, which worsens the actuator response as the motion speed increases. This time-dependent effect renders actuator control very challenging and, in practice, limits the functioning of this prototype to applications involving movements with limited dynamics (less than $0.5 \mathrm{~Hz}$, position cycles). For larger movement dynamics, different DE materials, such as silicone elastomers, should be employed.

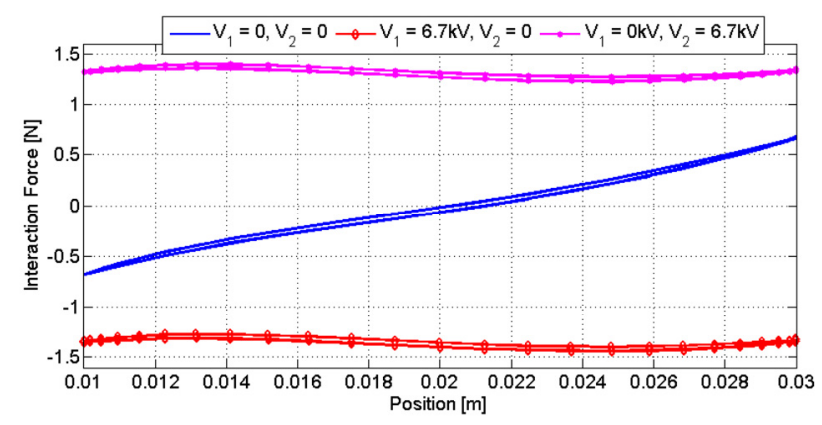

Figure 5: Actuator response ( $1 \mathrm{mHz}$ position cycle).

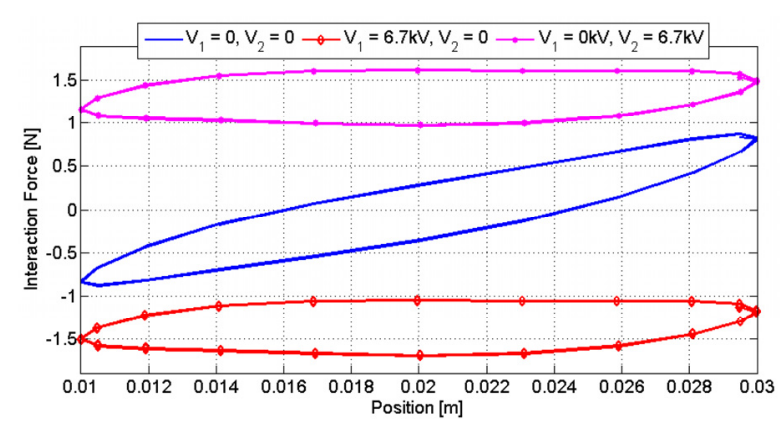

Figure 6: Actuator response (0.5 Hz position cycle). 


\section{CONTROL SYSTEM}

his section describes an interaction-force control system for the considered agonist-antagonist DE actuator. The controller is based on an optimum observer and on a suitable state-feedback law.

In particular, for controller design purposes and owing to simulation results reported in the previous section, the actuator model has been linearized such that the considered prototype can be described via the lumped parameter system depicted in Fig. 7, where $m(m=105 \mathrm{~g})$ is the effective inertia of the actuator output (comprising the sensor mass too), $K_{l}$ $\left(K_{l}=66.5 \mathrm{~N} / \mathrm{m}\right)$ is a constant stiffness coefficient capturing the OFF-state quasi-static linear elastic response of the actuator, $F_{\text {int }}$ is the interaction force exchanged with the environment, $F_{\text {dist }}$ is a disturbance force accounting for the unmodelled non-linear and time-dependent mechanical response of the system (comprising DE film visco-elasticity), and $F_{e m, l}$ is the "electric" force generated by the electrical activation of the agonist-antagonist DE films. Based on Eq. (2), for the considered DE actuator prototype

$$
F_{\text {em }, l}=K_{l}\left[(d-\delta)\left(\frac{V_{2}}{V_{\max }}\right)^{2}-(d+\delta)\left(\frac{V_{1}}{V_{\max }}\right)^{2}\right]
$$

where $V_{\max }\left(V_{\max }=6.7 \mathrm{kV}\right)$ is the maximum voltage which can be placed between each pair of DE actuator electrodes. As a result, the DE actuator dynamics can be written as

$$
F_{\text {int }}=m \ddot{\delta}+K_{l} \delta+F_{\text {dist }}+F_{\text {em }, l}
$$

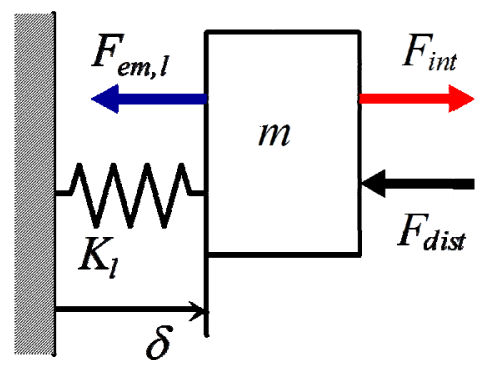

Figure 7: Actuator lumped-parameter model.

\section{Optimal Actuator State Estimator}

Controller development requires the complete knowledge of the variables $\delta$ (along with its time derivative), $F_{\text {int }}$ and $F_{d i s t}$ The considered actuator is equipped with a position and a force sensor that enable the straight measurement of $\delta$ and $F_{\text {int; }}$; however, no direct information is available for $F_{\text {dist. }}$ While this disturbance force could be determined via the visco-elastic model described before, for control purposes we have preferred to estimate it via a Kalman filter. Specifically, consider the augmented state-space system of the DE actuator dynamics

$$
\begin{aligned}
& \left\{\begin{array}{l}
\dot{\boldsymbol{x}}=\mathbf{A} \boldsymbol{x}+\mathbf{B} F_{\text {ele }}+\boldsymbol{\Gamma} \boldsymbol{u} \\
\boldsymbol{y}=\mathbf{C} \boldsymbol{x}+\boldsymbol{w}
\end{array}\right. \\
& \mathbf{A}=\left[\begin{array}{cccc}
0 & -K / m & -1 / m & 1 / m \\
1 & 0 & 0 & 0 \\
0 & 0 & 0 & 0 \\
0 & 0 & 0 & 0
\end{array}\right], \mathbf{B}=\left[\begin{array}{c}
-1 / m \\
0 \\
0 \\
0
\end{array}\right], \Gamma=\left[\begin{array}{ll}
0 & 0 \\
0 & 0 \\
0 & 1 \\
1 & 0
\end{array}\right], \boldsymbol{u}=\left[\begin{array}{l}
u_{1} \\
u_{2}
\end{array}\right], \mathbf{C}=\left[\begin{array}{ll}
0 & 0 \\
1 & 0 \\
0 & 0 \\
0 & 1
\end{array}\right]^{T}
\end{aligned}
$$

where

$$
\begin{aligned}
& \boldsymbol{x}=\left[\begin{array}{llll}
\dot{\delta} & \delta & F_{\text {dist }} & F_{\text {int }}
\end{array}\right]^{T} \\
& \boldsymbol{w}=\left[\begin{array}{ll}
w_{\text {pos }} & w_{\text {force }}
\end{array}\right]^{T}
\end{aligned}
$$

are respectively the state-space variable and the sensor noise vectors (with $W_{\text {force }}$ and $W_{\text {pos }}$ being the effective sensor variances), and $u_{1}$ and $u_{2}$ are white noise processes with variances $U_{1}$ and $U_{2}$. In the system, $F_{\text {dist }}$ and $F_{\text {int }}$ have been considered as Wiener processes [9], that is as continuous functions which vary slowly with independent increments (namely $\dot{F}_{\text {dist }} \approx u_{1}$ and $\dot{F}_{\text {int }} \approx u_{2}$ ). Then, the estimate 


$$
\hat{\boldsymbol{x}}=\left[\begin{array}{llll}
\hat{\dot{\delta}} \hat{\delta} & \hat{F}_{\text {dist }} & \hat{F}_{\text {int }}
\end{array}\right]^{T}
$$

of the state-space variable vector follows as

$$
\dot{\hat{\boldsymbol{x}}}=\mathbf{A} \hat{\boldsymbol{x}}+\mathbf{B} F_{e m, l}+\mathbf{L}(\boldsymbol{y}-\mathbf{C} \hat{\boldsymbol{x}})
$$

with $\mathbf{L}$ being the steady-state observer gain matrix which can be determined via the procedure described in [9] once the unknown observer parameters $U_{1}$ and $U_{2}$ are chosen.

\section{Actuator Controller}

For a given desired interaction force $F_{\text {int }}^{D}$, the following state-feedback law is chosen for the controller

$$
F_{\text {em }, l}^{C}=-K_{l} \hat{\delta}-\hat{F}_{\text {dist }}+F_{\text {int }}^{D}+G\left(F_{\text {int }}^{D}-\hat{F}_{\text {int }}\right)
$$

with $\mathrm{G}$ being the force error gain.

Then, among all the possible infinite choices granted by Eq. (13), the following activation laws have been chosen for the agonist and antagonist DE films

$$
\begin{aligned}
& V_{1}=V_{\max } \sqrt{\left(\left|F_{e m, l}^{C}\right|-F_{e m, l}^{C}\right) / 2 K_{l}(d+\delta)} \\
& V_{2}=V_{\max } \sqrt{\left(F_{e m, l}^{C}+\left|F_{e m, l}^{C}\right|\right) / 2 K_{l}(d-\delta)}
\end{aligned}
$$

Note that Eq. (18) implies reciprocal activation of the agonist-antagonist DE films. This is the simplest form of agonistantagonist actuator command.

\section{EXPERIMENTAL RESULTS}

$\mathrm{F}$ or validation purposes, a prototype of the agonist-antagonist DE actuator depicted in Fig. 1 has been embedded and tested in a properly designed test-bench as shown in Fig. 8. In the reported set-up, the DE actuator output is connected through a custom-made force sensor to a position-controlled linear brushless DC motor equipped with a built-in position sensor. In the experiments, these sensors are used to infer actuator interaction force $F_{\text {int }}$ and output position $\delta$ respectively. The employed force and position sensors are affected by white noise disturbances $w_{\text {force }}$ and $w_{\text {pos }}$ with variances equaling $W_{\text {forre }}=10 \mathrm{e}-5 \mathrm{~N}$ and $W_{\text {pos }}=8 \mathrm{e}-10 \mathrm{~m}$. As for the employed driving electronics, each of the agonist and antagonist DE films is activated by an efficient compact and low-cost electronic driver, which is custom-made and based on a two-transistor discontinuous-mode fly-back converter topology. Regarding specifications, the two considered drivers are capable of regulating their output voltages (namely $V_{1}$ and $V_{2}$ ) from 0 to $V_{\max }\left(V_{\max }=6.7 \mathrm{kV}\right.$ ) with a $12 \mathrm{~Hz}$, cut-off frequency. A picture of one of the driver prototypes is reported in Fig. 9; more details on driver design and performances can be found in [15].

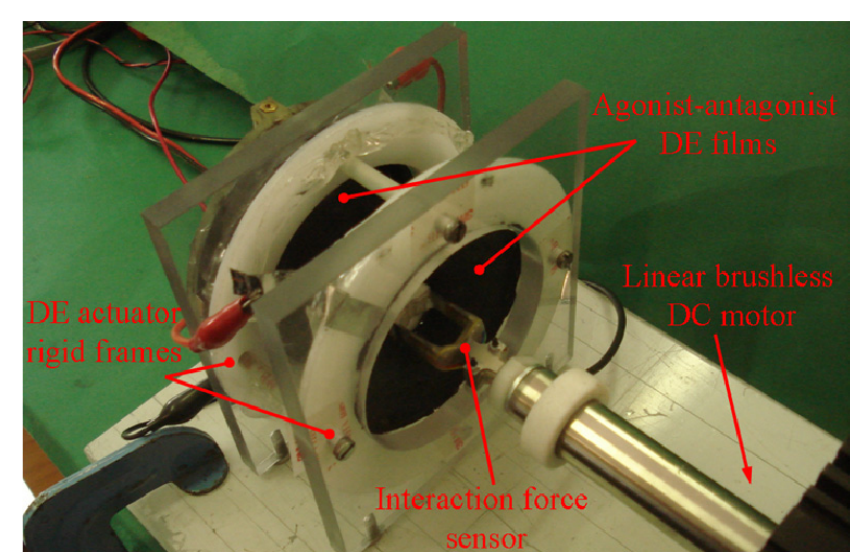

Figure 8: Actuator test-bench. 


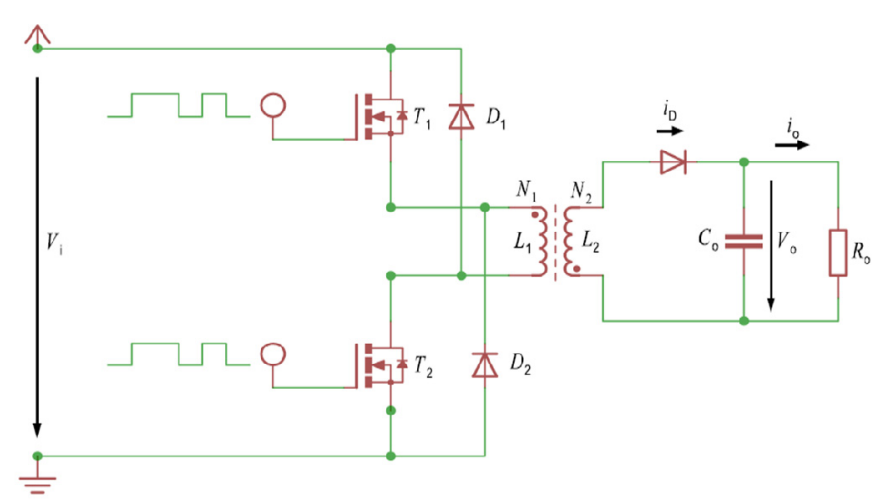

(a)

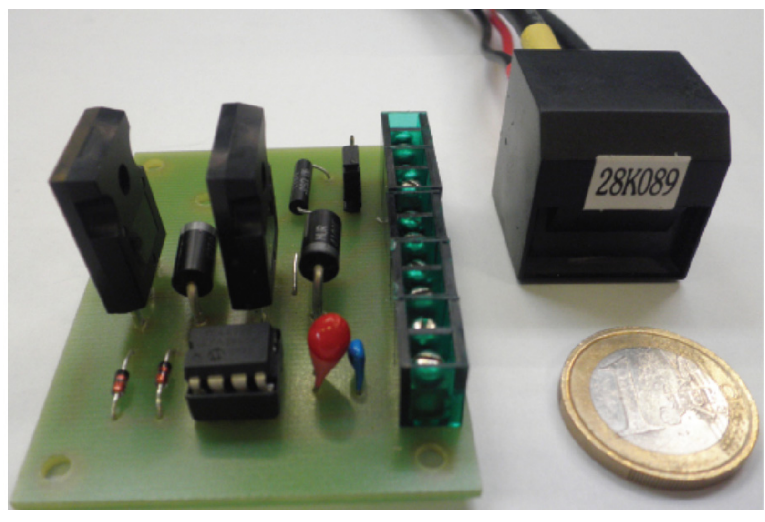

(b)

Figure 9: Custom made electronic driver prototype. (a) Circuit diagram; (b) PCB circuit board prototype.

In the experimental tests, the interaction force exchanged between DE actuator and DC motor is forced to follow a triangular interaction-force signal $F_{i n t}^{D}$, while the DC motor cyclically displaces the DE actuator output about its OFFstate rest position with a sinusoidal trajectory. The gain $G$ of the control law described by Eq. (17) is set to 3 . With the choice $U_{1}=U_{2}=10$, the steady-state observer (Kalman) matrix to be used in Eq. (16) is set to

$$
\mathbf{L}=\left[\begin{array}{cccc}
20363 & 201 & -111785 & 2 e 3 \\
8.29 & 0.016 & 5.6595 & 316
\end{array}\right]^{T}
$$

The experimental results for a desired interaction-force varying linearly between $\pm 1.5 \mathrm{~N}$ with frequency equalling $1 / 6 \mathrm{~Hz}$ and for a sinusoidal output motion varying between $\pm 10 \mathrm{~mm}$ with frequency equalling $0.5 \mathrm{~Hz}$ are reported in Fig. 10 .
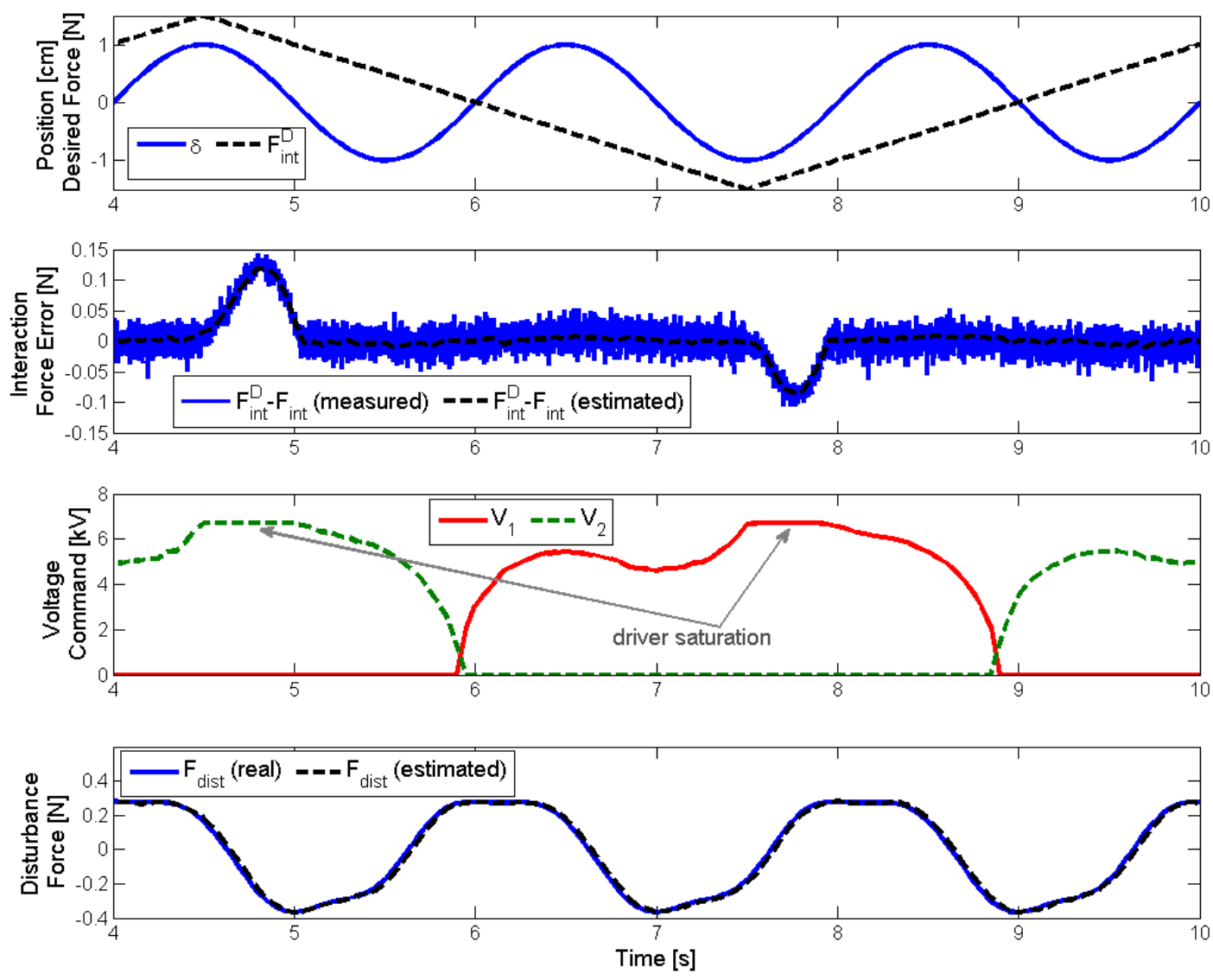

Figure 10: DE actuator force-controller validation. 
Imposed output motion and desired interaction force command are shown in the first (upper) plot. Measured and estimated interaction force tracking errors are shown in the second plot. The reciprocal DE actuator activation voltages computed via Eq. (18) are reported in the third plot. Real (a-priori measured) and estimated (via Eq. (16)) disturbance forces are shown in the fourth plot. In particular, the second plot highlights that, for a large part of the experiment duration, the proposed controller is able to keep the measured interaction-force tracking error within $\pm 0.05 \mathrm{~N}$, which is roughly equal to the force sensor measurement noise. Problems occur between the time intervals spanning from $4.4 \mathrm{~s}$ and 5.1s and from 7.5 and 8 s which, as the third plot shows, are only due to the saturation of the voltage commands $V_{2}$ and $V_{1}$ (respectively) rather than to a fault of the controller. Note indeed from Fig. 6 that, because of the large hysteresis affecting the employed DE films at the imposed motion frequency, the considered agonist-antagonist DE actuator cannot generate forces greater than $1 \mathrm{~N}$ for large part of its backward stroke (forces greater than $1.5 \mathrm{~N}$ can instead be produced for large part of its forward stroke).

\section{CONCLUSIONS}

I $\mathrm{n}$ this paper, a closed-loop interaction-force controller for an agonist-antagonist linear actuator based on conicallyshaped Dielectric Elastomer films has been proposed and validated. The system represents a first step towards the production of practical DE-based force feedback devices and HI. At first, a model accounting for the viscohyperelastic nature of the DE films has been presented for actuator electro-mechanical design purposes. The model was then linearized and employed for controller synthesis purposes. The developed controller requires a position sensor and a force sensor, implements a reciprocal activation strategy of the agonist-antagonist Dielectric Elastomer films, employs a state-feedback control law and features a Kalman filter which, beside reducing the measurement noise, enables accurate estimation of the dynamic viscous response of the actuator. Experimental results showed that the proposed interactionforce controller possesses good force tracking performance whose accuracy is comparable to that of the employed force sensor. Due to the significant hysteretic response of the adopted elastomeric material, the force generating ability of the proposed actuator-controller system demonstrated to be valid only for interaction applications involving movements with small-to-medium dynamics. In case higher speeds of motion are required, different DE materials such as silicone elastomers can be used.

\section{REFERENCES}

[1] R. Pelrine, R. Kornbluh, J. Joseph, Sensors Actuators A, 64(1) (1998) 77.

[2] R. Vertechy, G. Berselli, V. Parenti Castelli, G. Vassura, Journal of Intelligent Material Systems and Structures, DOI: 10.1177/1045389X09356608, 21(5) (2010) 503.

[3] R. Vertechy, G. Berselli M. Bergamasco, V. Parenti Castelli, In: Advances in Robot Kinematics: Motion in Man and Machine, J. Lenarcic and M. Stanisic Eds., Springer, DOI: 10.1007/978-90-481-9262-5_14, Dordrecht, the Netherlands, (2010) 127.

[4] F. Carpi, G. Frediani, D. De Rossi, IEEE Trans. on Biomedical Engineering, 56(9) (2009) 2327.

[5] M. Y. Ozsecen, M. Sivak, C. Mavroidis, In: Proc. of SPIE, 7647 (2010) 764737(7).

[6] Berselli, R. Vertechy, G. Vassura, V. Parenti Castelli, IEEE Transactions on Mechatronics, DOI: 10.1109/TMECH.2010.2090664, 16(1) (2011) 67.

[7] Y. C. Fung, Biomechanics: Mechanical Properties of Living Tissues, Springer-Verlag, Berlin, (1993).

[8] G. Berselli, R. Vertechy, M. Babič, V. Parenti Castelli, Journal of Intelligent Material Systems and Structures, DOI: 10.1177/1045389X12457251, first published on August 28 (2012).

[9] B. Friedland, Control System Design: An Introduction to State Space Methods. Dover Publications, New York, (2005).

[10] L. Howell, Compliant Mechanisms, John Wiley and Sons, New York, (2001).

[11] G. Kofod, “The static actuation of dielectric elastomer actuators: how does pre-stretch improve actuation?”. J. Phys. D: Appl. Phys., vol. 41, pp. 215405(11), 2008.

[12] J. S. Plante, S. Dubowsky, Smart Materials and Structures, 16(2) (2007) 227.

[13] W. N. Findley, J. S. Lai, K. Onaran, Creep and relaxation of nonlinear viscoelastic materials: with an introduction to linear Viscoelasticity. Dover pubblications, New York, (1989).

[14] O. H. Yeoh, Rubber Chemistry and Technology, 63 (1990) 792. 
[15] M. Babic, R. Vertechy, G. Berselli, J. Lenarcic, V. Parenti Castelli, G. Vassura, Mechatronics, DOI: 10.1016/j.mechatronics.2009.11.006, 20(2) (2010) 201. 